\title{
Ocorrência de arritmias ventriculares e supraventriculares de acordo com o perfil clínico de pacientes renais crônicos em hemodiálise: estudo no Real Hospital
}

\section{Português de Pernambuco}

\author{
Occurrence of ventricular and supraventricular arrhythmias according to the clinical profile of \\ chronic renal patients undergoing hemodialysis: study at the Real Hospital Português de
}

Pernambuco

Aparición de arritmias ventriculares y supraventriculares según el perfil clínico de los pacientes renales crónicos en hemodiálisis: un estudio en el Real Hospital Português de Pernambuco

Recebido: 24/09/2021 | Revisado: 30/09/2021 | Aceito: 02/10/2021 | Publicado: 04/10/2021

\author{
Clarissa Jacob de Barros Carvalho \\ ORCID: https://orcid.org/0000-0001-7165-144X \\ Centro Universitário Faculdade de Medicina do ABC, Brasil \\ E-mail: clarissa.jbcarvalho@hotmail.com \\ Janilson José da Silva Júnior \\ ORCID: https://orcid.org/0000-0002-1843-0864 \\ Centro Universitário Maurício de Nassau, Brasil \\ E-mail: janilson_jose@ hotmail.com \\ Marcelo Rodrigues Bacci \\ ORCID: https://orcid.org/0000-0001-8578-8404 \\ Centro Universitário Faculdade de Medicina do ABC, Brasil \\ E-mail: marcelo.bacci@fmabc.br
}

\begin{abstract}
Resumo
Introdução: Nos últimos anos, a Doença Renal Crônica tem sido considerada um grave problema de saúde pública. Isso se deve principalmente à elevada mortalidade anual, devido a distúrbios cardiovasculares. Alguns dos principais responsáveis pela mortalidade dessa população são as doenças cardíacas e dentro delas os episódios arrítmicos. Diante dessa perspectiva, o presente estudo objetivou investigar a prevalência de arritmias ventriculares e supraventriculares em pacientes portadores de doença renal crônica dialítica. Método: Trata-se de um estudo de coorte prospectivo, no qual foi avaliada a presença de arritmias cardíacas e risco de morte súbita cardíaca em 35 pacientes do Real Hospital Português de Pernambuco. Resultados: Foi observado que 71,4\% dos pacientes são do sexo masculino, 85,7\% não é de cor negra e $57,1 \%$ possui idade acima de 50 anos. Pacientes com níveis plasmáticos inadequados de potássio, cálcio e aqueles que não utilizavam medicamentos, apresentaram maior prevalência de arritmias ventriculares e supraventriculares. Discussão: Alterações dos níveis normais de cálcio e potássio geram intercorrências cardiovasculares, principalmente, arritmias. Maior prevalência de arritmias em indivíduos sem uso de medicamentos pode estar relacionado pelo fato de muitos pacientes serem subdiagnosticados. Além disso, houve maior prevalência de arritmia ventricular durante a hemodiálise, quando comparada à arritmia supraventricular. A idade mostrou-se um importante fator relacionado às arritmias em pacientes dialíticos. Conclusão: Constata-se que pacientes renais crônicos dialíticos apresentam alterações dos níveis normais de eletrólitos e hipertensão arterial que aumentam a ocorrência de arritmias cardíacas, principalmente ventriculares, aumentando o risco de morte súbita nessa população. Palavras-chave: Doença renal; Arritmias; Morte súbita; Hemodiálise.
\end{abstract}

\begin{abstract}
Introduction: In recent years, Chronic Kidney Disease has been considered a serious public health problem. This is mainly due to the high annual mortality, due to cardiovascular disorders. Some of the main factors responsible for the mortality of this population are arrhythmic events. Given this perspective, the present study aimed to investigate the prevalence of ventricular and supraventricular arrhythmias in patients with dialysis chronic kidney disease. This is a prospective cohort study, in which the presence of cardiac arrhythmias and risk of sudden cardiac death was evaluated in 35 patients at Real Hospital Português de Pernambuco. Results: It was observed that $71.4 \%$ of the patients are male, $85.7 \%$ are not black and $57.1 \%$ are over 50 years old. Patients with inadequate plasma levels of potassium, calcium and those who did not use medications, had a higher prevalence of ventricular and supraventricular arrhythmias. Discussion: Changes in normal levels of calcium and potassium generate cardiovascular complications, especially arrhythmias. Higher prevalence of arrhythmias in individuals without medication use may be related to the fact that
\end{abstract}


many patients are underdiagnosed. In addition, there was a higher prevalence of ventricular arrhythmia during hemodialysis when compared to supraventricular arrhythmia. Age proved to be an important factor related to arrhythmias in dialysis patients. Conclusion: Chronic renal patients on dialysis are found to have alterations in normal electrolyte levels and arterial hypertension that increase the occurrence of cardiac arrhythmias, especially ventricular, increasing the risk of sudden death in this population.

Keywords: Kidney disease; Arrhythmias; Sudden death; Hemodialysis.

\section{Resumen}

Introducción: En los últimos años, la Enfermedad Renal Crónica se ha considerado un grave problema de salud pública. Esto se debe principalmente a la alta mortalidad anual por trastornos cardiovasculares. Algunas de las principales causas de mortalidad en esta población son las cardiopatías y, dentro de ellas, los episodios arrítmicos. Desde esta perspectiva, el presente estudio tuvo como objetivo investigar la prevalencia de arritmias ventriculares y supraventriculares en pacientes con enfermedad renal crónica en diálisis. Método: Se trata de un estudio de cohorte prospectivo, que evaluó la presencia de arritmias cardíacas y el riesgo de muerte súbita cardíaca en 35 pacientes del Real Hospital Português de Pernambuco. Resultados: Se observó que el 71,4\% de los pacientes son varones, el 85,7\% no son negros y el $57,1 \%$ son mayores de 50 años. Los pacientes con niveles plasmáticos inadecuados de potasio, calcio y los que no usaban medicación tenían una mayor prevalencia de arritmias ventriculares y supraventriculares. Discusión: Los cambios en los niveles normales de calcio y potasio generan complicaciones cardiovasculares, especialmente arritmias. La mayor prevalencia de arritmias en individuos sin medicación puede estar relacionada con el hecho de que muchos pacientes están infradiagnosticados. Además, hubo una mayor prevalencia de arritmia ventricular durante la hemodiálisis en comparación con la arritmia supraventricular. Se demostró que la edad es un factor importante relacionado con las arritmias en los pacientes en diálisis. Conclusión: Se comprueba que los pacientes renales crónicos en diálisis presentan alteraciones en los niveles normales de electrolitos e hipertensión arterial que aumentan la aparición de arritmias cardíacas, principalmente ventriculares, aumentando el riesgo de muerte súbita en esta población.

Palabras clave: Enfermedad renal; Arritmias; Muerte súbita; Hemodiálisis.

\section{Introdução}

Nos últimos anos, a Doença Renal Crônica (DRC) tem evoluído como um problema a nível global de saúde pública, afetando cerca de 10 a $15 \%$ da população adulta mundial. Diversos fatores contribuem com esse cenário, entre os quais, destacam-se a hipertensão arterial sistêmica, Diabetes Mellitus, obesidade e a maior longevidade da população (Collins et al., 2015).

No Brasil, estimativas acerca da prevalência da DRC são incertas, devido às limitações e heterogeneidade dos dados. Apesar disso, dados do Censo Brasileiro de Diálise apontam que mais de 6 milhões de brasileiros apresentem essa enfermidade (SBN, 2021). Desses, em 2018, mais de 130 mil recebiam terapia dialítica crônica. O avanço no número de pacientes com DRC torna o Brasil o país com o terceiro maior número de pacientes em tratamento dialítico. Dados de prevalência para essa doença são relevantes por subsidiarem o melhor planejamento de ações preventivas e assistenciais, além de contribuírem com a diminuição da morbimortalidade associada à DRC (Neves et al., 2020, Almeida et al., 2020; Marinho et al., 2020)

A DRC pode ser caracterizada pela alteração permanente da Taxa de Filtração Glomerular (TFG) por 12 semanas ou mais. Segundo o KDIGO, é recomendada a confirmação diagnóstica da DRC em adultos com TFG estimada (TFGe) entre 45$59 \mathrm{~mL} / \mathrm{min} / 1,73 \mathrm{~m} 2$, persistente por mais três meses (Bauer et al., 2008; Johnson et al., 2013; KDIGO, 2013).

A doença renal agrava a hipertrofia ventricular, a aterosclerose, causando complicações cardiovasculares, além de sustentar o estado inflamatório crônico, hipervolemia, anemia, desnutrição e distúrbios no metabolismo mineral, como hipercalcemia e hiperfosfatemia (Bonato \& Canziani, 2017).

A hipercalcemia pode causar calcificação das artérias coronárias, aumentando o risco cardiovascular. Além disso, distúrbios envolvendo o cálcio e o fosfato desencadeiam aumento da vulnerabilidade à pós-despolarização precoce, fibrose cardíaca e arritmias ventriculares, aumentando o risco de morte súbita. Nos pacientes em terapia dialítica, esses fatores aumentam em 20 vezes as chances de eventos cardiovasculares fatais (Hsueh et al., 2014; Donfrancesco et al., 2013).

As doenças cardiovasculares mais prevalentes em pacientes com DRC em estágio dialítico são insuficiência cardíaca 
congestiva, doença coronariana e arritmias cardíacas. Além disso, a principal causa morte entre os pacientes com DRC em terapia dialítica, especialmente hemodiálise, está associada a distúrbios do ritmo cardíaco. A maior susceptibilidade para arritmias ventriculares em pacientes com DRC relaciona-se a alterações da estrutura cardíaca e alterações metabólicas (Bonato \& Canziani, 2017).

A morte súbita cardíaca é responsável por aproximadamente $60 \%$ da mortalidade cardiovascular nos pacientes renais crônicos em terapia dialítica (Bignotto et al., 2012).

As arritmias cardíacas são algumas das maiores condições responsáveis pelos desfechos fatais nesses casos, especialmente as arritmias ventriculares e supraventriculares, também conhecidas como taquicardia supraventricular. Outros fatores estão relacionados com o aumento do risco de morte súbita cardíaca nos pacientes com DRC em hemodiálise como: Diabetes Mellitus, hipertensão arterial sistêmica (HAS), insuficiência cardíaca congestiva (ICC) e apneia obstrutiva do sono (Verde et al., 2016; Lullo et al., 2016).

Diante dessa perspectiva, o presente estudo tem como objetivo descrever a prevalência e os tipos de arritmias cardíacas em pacientes portadores de doença renal crônica em hemodiálise e os fatores que podem interferir na sua ocorrência.

\section{Metodologia}

Trata-se de um estudo de coorte prospectivo, de cunho transversal, qual foram analisados pacientes renais crônicos dialíticos num período de um ano (Janeiro a Dezembro de 2020) quanto à presença de arritmias cardíacas e risco de morte súbita cardíaca. Foram incluídos no estudo pacientes com DRC em hemodiálise e idade maior ou igual a 18 anos e excluídos pacientes com neoplasia e transplantados renais, portadores de marcapasso e paciente com cardiodesfibrilador implantado. Os pacientes incluídos no estudo faziam tratamento de hemodiálise há pelo menos 3 meses. Após aprovação pelo Comitê de Ética em Pesquisa da Plataforma Brasil, o estudo foi realizado no Serviço de Nefrologia do Real Hospital Português de Pernambuco (RHP). Todos os parâmetros só foram coletados após o consentimento dos pacientes.

Os pacientes foram submetidos a exame físico e avaliação clínica apenas durante a sessão de hemodiálise. Os pacientes preencheram protocolo de estudo composto por dados demográficos, socioeconômicos, antropométricos, clínicos e laboratoriais. Os exames laboratoriais, como níveis plasmáticos de cálcio, fósforo, magnésio e potássio, foram coletados durante o período pré e pós hemodiálise. Além disso, foram analisados dados eletrocardiográficos desses pacientes através do exame Holter de 24 horas, de modo a correlacionar a condição renal e eventos cardiovasculares, como arritmias e risco de morte súbita cardíaca. O Holter de 24 horas foi instalado imediatamente antes do início da sessão de hemodiálise. Dados demográficos, socioeconômicos e clínicos foram obtidos por meio de prontuário médico e avaliação clínica. Dados laboratoriais foram adquiridos pela análise de amostras de sangue arterial obtidos no início e ao final da sessão de hemodiálise.

O diagnóstico das arritmias foi realizado por meio de monitorização eletrocardiográfica contínua através de gravação digital por Holter Cardio Light ${ }^{\circledR}$ de três canais da Cardios (24h), instalado no início da sessão de hemodiálise e mantido por 24 horas. Esse dispositivo de monitorização contínua possibilita a realização de registros eletrocardiográficos antes, durante e após a sessão hemodialítica. As informações foram registradas em software específico do dispositivo, CardioNet (Holter), que foi interpretado pelo mesmo examinador e sob a mesma forma para todas os indivíduos. Os laudos descritivos do dispositivo foram obtidos através de servidor de análise verificados por um profissional especialista em eletrocardiografia. A interpretação dos resultados do Holter anormal resulta da observação do ritmo cardíaco irregular, fora do padrão, cuja normalidade em geral está entre 50 e 100 batimentos por minuto. Duas arritmias foram consideradas nas análises: arritmia ventricular e arritmia supraventricular (taquicardia supraventricular).

A análise estatística foi realizada por meio de tabulação de dados em planilha eletrônica Microsoft Excel e submissão ao software SPSS, versão 18 (SPSS, 2009). Para caracterizar o perfil pessoal clínico dos pacientes avaliados, foram calculadas 
as frequências percentuais e construídas as respectivas distribuições de frequência. Foi aplicado o teste Qui-quadrado para comparar os percentuais encontrados nas categorias das variáveis, de modo a promover uma comparação de proporção.

Foi construída uma tabela de contingência para verificar a influência do nível de potássio, cálcio, ultrafiltração (UF) e o uso de medicamento que interferem no ritmo cardíaco como beta-bloqueadores e bloqueadores de canais de cálcio não diidropiridínicos na ocorrência de arritmias ventriculares e supra-ventriculares. Foram avaliados os níveis de potássio $(\mathrm{K})$ préhemodiálise e a diferença entre o nível de potássio antes e após a hemodiálise $(\Delta \mathrm{K})$. Foi avaliada a diferença entre o nível de cálcio antes e após a hemodiálise $(\Delta \mathrm{Ca})$. Foi Avaliada dose da diálise $(\mathrm{Kt} / \mathrm{V})$, que está relacionada à qualidade da diálise. Foi avaliada também a UF e correlacionados todos esses parâmetros com a ocorrência de arritmia ventricular ou supraventricular.

Foi realizada uma análise bivariada, que visou:

- Verificar se os pacientes com potássio (K) pré-hemodiálise $\geq 4,5$ e Delta K (diferença entre o nível de potássio antes e após a hemodiálise) $\geq 1,3$, se tiveram mais arritmias que os demais e se as arritimas eram ventriculares ou Supraventriculares;

- Verificar se os pacientes com cálcio Delta Ca (diferença entre o nível de cálcio antes e após a hemodiálise) $\geq 1,2$, se tiveram mais Arritmias Ventriculares ou Arritmias Supra-ventriculares, bem como mais arritmias que os demais;

- Verificar se os pacientes UF $(\mathrm{Kg} / \mathrm{h}) \geq 0,8$, se tiveram mais arritmias do que os demais pacientes.

- Verificar se os pacientes que faziam medicação que interferem no ritmo cardíaco como beta-bloqueadores e bloqueadores de canais de cálcio não diidropiridínicos (código 1) tiveram mais arritmias do que os demais pacientes, bem como avaliar se a arritmia era ventricular ou supraventricular.

Para avaliar a associação destes fatores com a arritmia foi aplicado o teste Qui-quadrado para independência. Nos casos em que as suposições do teste Qui-quadrado não foram satisfatórias, aplicou-se o teste Exato de Fisher. Nos casos que foi encontrada associação, foi calculada a razão de prevalência para comparar os riscos ao desenvolvimento de arritmias, entre as categorias avaliadas. Todas as conclusões foram tiradas considerando o nível de significância de 5\%.

\section{Resultados e Discussão}

Foi gerada uma população amostral de 35 indivíduos, conforme o fluxograma da Figura 1. Também estão dispostos na Figura 1 o tempo que os pacientes incluídos no estudo estavam em terapia renal substitutiva (hemodiálise). 
Figura 1. População amostral.

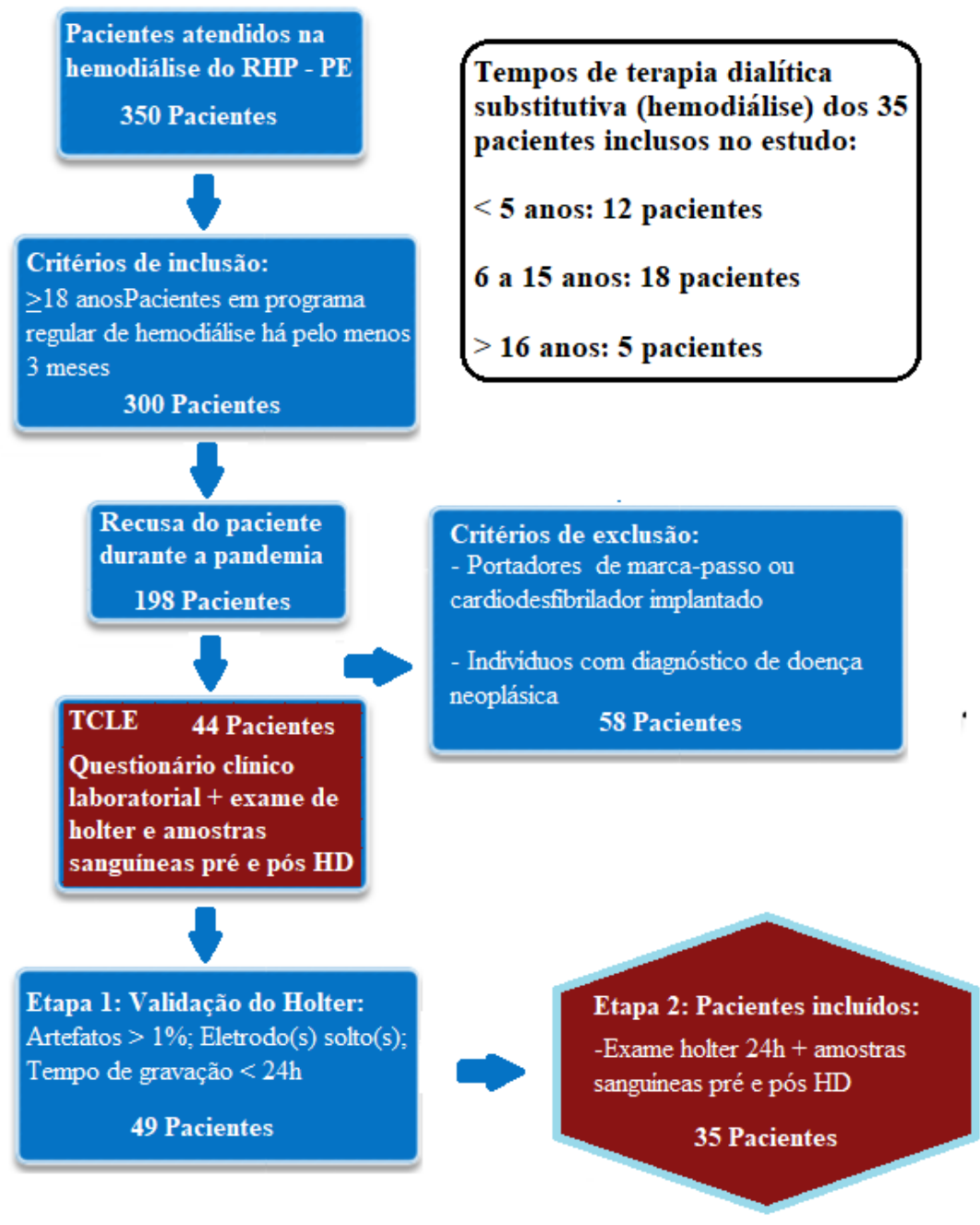

Fonte: Autores (2021).

Na Tabela 1 temos a distribuição do perfil pessoal dos pacientes avaliados. Verifica-se que a maioria é do sexo masculino $(71,4 \%)$ possui idade de 30 a 49 anos $(42,9 \%)$ e não é da cor negra $(85,7 \%)$. O teste de comparação de proporção foi significativo para o sexo ( $\mathrm{p}$-valor $=0,011)$ e para a etnia (p-valor $<0,001)$, indicando que o grupo de homens e não negros é relevantemente maior entre os pacientes avaliados.

Também está disposto na Tabela 1 o perfil clínico dos pacientes avaliados. Verifica-se que a maioria não possui DM (77,1\%), apresenta HAS (77,1\%), não tem ICC (74,3\%), não apresenta HVE (80,0\%), possui etiologia DRC desconhecida $(34,3 \%)$, está com IMC normal $(57,1 \%)$, apresenta normalidade da albumina $(97,1 \%)$, apresenta normalidade na Upré $(77,1 \%)$ e teve Kt/V eficiente $(94,3 \%)$. 
Tabela 1. Distribuição do perfil pessoal e do perfil clínico dos pacientes avaliados. Valores normais de Albumina (3,5g/dL e $5,0 \mathrm{~g} / \mathrm{dL}) ; \mathrm{Kt} / \mathrm{V}>1,2$.

\begin{tabular}{|c|c|c|}
\hline Fator avaliado & $\mathrm{n}$ & $\%$ \\
\hline \multicolumn{3}{|l|}{ Sexo } \\
\hline Masculino & 25 & 71,4 \\
\hline Feminino & 10 & 28,6 \\
\hline \multicolumn{3}{|l|}{ Idade } \\
\hline 30 a 49 anos & 15 & 42,9 \\
\hline 50 a 59 anos & 10 & 28,6 \\
\hline 60 anos ou mais & 10 & 28,6 \\
\hline Mínimo-máximo & $30,0-70,0$ & \\
\hline Média \pm Desvio padrão & $52,9 \pm 10,0$ & \\
\hline \multicolumn{3}{|l|}{ Etnia } \\
\hline Não negro & 30 & 85,7 \\
\hline Negro & 5 & 14,3 \\
\hline \multicolumn{3}{|l|}{ Diabetes Mellitus (DM) } \\
\hline Não & 27 & 77,1 \\
\hline Sim, possui DM & 8 & 22,9 \\
\hline \multicolumn{3}{|l|}{ Hipertensão Arterial Sistêmica (HAS) } \\
\hline Não & 8 & 22,9 \\
\hline Sim, possui HAS & 27 & 77,1 \\
\hline \multicolumn{3}{|l|}{ Insuficiência Cardíaca Congestiva (ICC) } \\
\hline Não & 26 & 74,3 \\
\hline Sim, possui ICC & 9 & 25,7 \\
\hline \multicolumn{3}{|l|}{ Hipertrofia Ventricular Esquerda (HVE) } \\
\hline Não & 28 & 80,0 \\
\hline Sim, possui HVE & 7 & 20,0 \\
\hline \multicolumn{3}{|l|}{ Etiologia DRc } \\
\hline HAS & 9 & 25,7 \\
\hline $\mathrm{DM}$ & 4 & 11,4 \\
\hline $\begin{array}{l}\text { Doença Renal Policística Autossômica Dominante } \\
\text { (DRPAD) }\end{array}$ & 4 & 11,4 \\
\hline Glomerulopatia & 4 & 11,4 \\
\hline HIV & 1 & 2,9 \\
\hline Uropatia Obstrutiva & 1 & 2,9 \\
\hline Indeterminada & 12 & 34,3 \\
\hline \multicolumn{3}{|l|}{ Índice de Massa Corpórea (IMC) } \\
\hline Baixo peso & 1 & 2,9 \\
\hline Normal & 20 & 57,1 \\
\hline Sobrepeso & 6 & 17,1 \\
\hline Obeso & 8 & 22,9 \\
\hline \multicolumn{3}{|l|}{ Albumina (g/dL) } \\
\hline Anormal $(<3,5)$ & 1 & 2,9 \\
\hline Normal $(\geq 3,5)$ & 34 & 97,1 \\
\hline \multicolumn{3}{|l|}{$\mathrm{Kt} / \mathrm{V}$} \\
\hline Não eficiente $(<1,2)$ & 2 & 5,7 \\
\hline Eficiente $(\geq 1,2)$ & 33 & 94,3 \\
\hline
\end{tabular}

Fonte: Autores (2021).

A variável idade foi significativa $(\mathrm{p}=0.008)$ para as arritmias supraventriculares. A Figura 2 ilustra a probabilidade de ocorrência de arritmias supraventriculares em função da idade. Trata-se da distribuição desse tipo de arritmia em função da idade dos indivíduos. $\mathrm{O}$ aumento da idade dos indivíduos aumenta a probabilidade da ocorrência de arritmia. 
Figura 2. Curva de probabilidade de arritmias supraventriculares como função da idade. Dados analisados no R Core Team (2013).

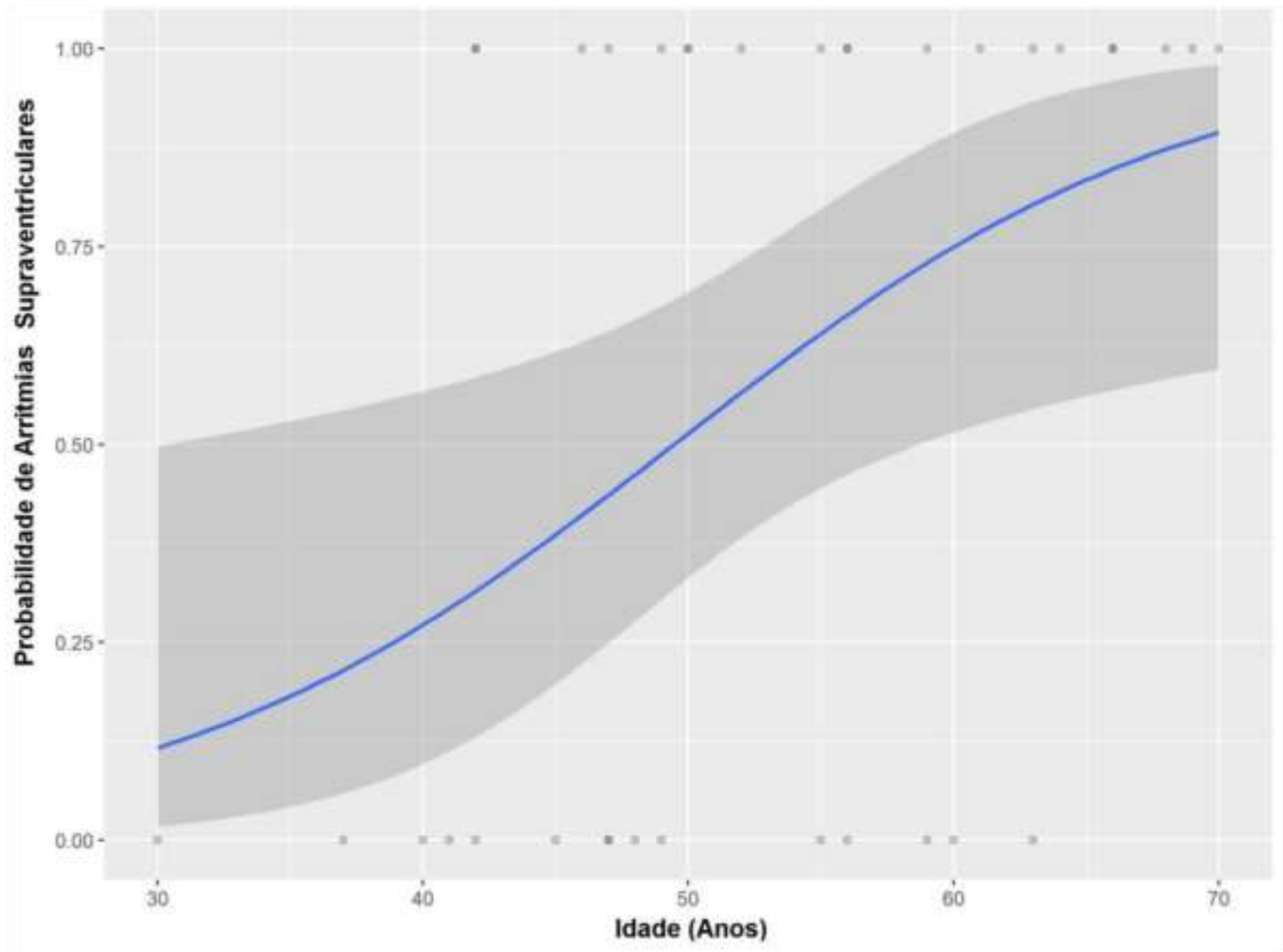

Fonte: Autores (2021).

Para o modelo de regressão logística combinando as variáveis duas a duas, o efeito combinado de delta K com a idade $(\mathrm{p}=0,008)$ e de delta $\mathrm{Ca}$ com a idade $(\mathrm{p}=0,011)$ são estatisticamente significativos para as arritmias supraventriculares (Tabela 2).

Tabela 2. Modelo de regressão logística combinando duas variáveis para arritmias supraventriculares.

\begin{tabular}{|c|c|c|c|c|c|c|c|c|c|c|}
\hline & $\begin{array}{c}\text { Delta } \\
\text { CA }\end{array}$ & Idade & Sexo & Etnia & HAS & $\mathbf{K t} / \mathbf{V}$ & Tempo HD & IMC & Peso Seco & Medicações \\
\hline Delta K & 0,155 & 0,008 & 0,208 & 0,789 & 0,202 & 0,849 & 0,716 & 0,718 & 0,915 & 0,160 \\
\hline Delta CA & & 0,011 & 0,142 & 0,887 & 0,200 & 0,838 & 0,779 & 0,644 & 0,886 & 0,207 \\
\hline Idade & & & 0,355 & 0,852 & 0,316 & 0,850 & 0,463 & 0,799 & 0,899 & 0,153 \\
\hline Sexo & & & & 0,968 & 0,243 & 0,952 & 0,688 & 0,443 & 0,990 & 0,264 \\
\hline Etnia & & & & & 0,202 & 0,800 & 0,726 & 0,707 & 0,931 & 0,163 \\
\hline HAS & & & & & & 0,698 & 0,360 & 0,728 & 0,780 & 0,092 \\
\hline Ktv & & & & & & & 0,719 & 0,581 & 0,930 & 0,165 \\
\hline Tempo HD & & & & & & & & 0,874 & 0,688 & 0,259 \\
\hline IMC & & & & & & & & & 0,395 & 0,161 \\
\hline Peso Seco & & & & & & & & & & 0,163 \\
\hline
\end{tabular}


Na Tabela 3 temos a distribuição da arritmia ventricular e arritmia supraventricular segundo a avaliação do potássio, cálcio, UF e uso de medicamentos. Verifica-se maior prevalência da arritmia ventricular nos pacientes com risco no nível de potássio $(53,8 \%)$, sem risco no nível de Ca $(66,7 \%)$, com risco no nível do UF $(71,4 \%)$ e que não utiliza medicamento $(53,8 \%)$.

Tabela 3. Distribuição da arritmia ventricular e arritmia supra-ventricular segundo a avaliação do potássio, cálcio, UF e uso de medicamentos.

\begin{tabular}{|c|c|c|c|c|}
\hline \multirow{3}{*}{ Fator avaliado } & \multicolumn{4}{|c|}{ Tipo de arritmia } \\
\hline & \multicolumn{2}{|c|}{$\begin{array}{c}\text { Arritmias } \\
\text { Ventriculares }\end{array}$} & \multicolumn{2}{|c|}{$\begin{array}{c}\text { Arritmias } \\
\text { Supra-ventriculares }\end{array}$} \\
\hline & Sim & Não & Sim & Não \\
\hline $\begin{array}{l}\text { Avaliação do } \mathbf{K}(\mathbf{m m o l} / \mathbf{L}) \\
\text { Não risco } \\
\text { Risco }(K \text { pre } \geq 4,5 \text { e } \Delta K \geq 1,3)\end{array}$ & $\begin{array}{c}11(50,0 \%) \\
7(53,8 \%)\end{array}$ & $\begin{array}{c}11(50,0 \%) \\
6(46,2 \%) \\
\end{array}$ & $\begin{array}{l}10(45,5 \%) \\
10(76,9 \%) \\
\end{array}$ & $\begin{array}{c}12(54,5 \%) \\
3(23,1 \%)\end{array}$ \\
\hline $\begin{array}{l}\text { Avaliação do Ca }(\mathbf{m m o l} / \mathbf{L}) \\
\text { Não risco } \\
\operatorname{Risco}(\Delta \mathrm{Ca} \geq 1,2)\end{array}$ & $\begin{array}{c}12(66,7 \%) \\
6(35,3 \%)\end{array}$ & $\begin{array}{c}6(33,3 \%) \\
11(64,7 \%)\end{array}$ & $\begin{array}{c}11(61,1 \%) \\
9(52,9 \%)\end{array}$ & $\begin{array}{l}7(38,9 \%) \\
8(47,1 \%)\end{array}$ \\
\hline $\begin{array}{l}\text { Avaliação do UF (L/dia) } \\
\text { Não risco } \\
\text { Risco (UF } \geq 0,8)\end{array}$ & $\begin{array}{c}13(46,4 \%) \\
5(71,4 \%)\end{array}$ & $\begin{array}{c}15(53,6 \%) \\
2(28,6 \%)\end{array}$ & $\begin{array}{c}16(57,1 \%) \\
4(57,1 \%)\end{array}$ & $\begin{array}{c}12(42,9 \%) \\
3(42,9 \%)\end{array}$ \\
\hline $\begin{array}{l}\text { Uso de medicamento } \\
\text { Não } \\
\text { Sim }\end{array}$ & $\begin{array}{c}14(53,8 \%) \\
4(44,4 \%)\end{array}$ & $\begin{array}{c}12(46,2 \%) \\
5(55,6 \%)\end{array}$ & $\begin{array}{l}18(69,2 \%) \\
2(22,2 \%)\end{array}$ & $\begin{array}{l}8(30,8 \%) \\
7(77,8 \%)\end{array}$ \\
\hline
\end{tabular}

${ }^{1} \mathrm{p}$-valor do teste qui-quadrado para independência

${ }^{2}$ p-valor do teste Exato de Fisher.

Valores normais: $\mathrm{K}(3,5-5,5 \mathrm{mmol} / \mathrm{L})$; $\mathrm{Ca}(2,1-2,5 \mathrm{mmol} / \mathrm{L})$

Fonte: Autores (2021).

Embora seja encontrada maior prevalência de arritmia ventricular no grupo de pacientes com o perfil descrito, o teste de independência não foi significativo para os fatores avaliados. Apesar disso, observa-se uma tendência de pacientes com níveis anormais de potássio, cálcio e UF e sem uso de medicamento apresentarem arritmias ventriculares.

Para arritmia supraventricular, verifica-se maior prevalência no grupo de pacientes com risco no nível de potássio (76,9\%), sem risco no nível de Ca $(61,1 \%)$ e que não utiliza medicação $(69,2 \%)$. Para avaliação do UF a prevalência de arritmias supraventricular foi semelhante no grupo que possui risco e que não possui risco (ambos grupos com 57,1\%) embora seja encontrada maior prevalência de arritmia supraventricular no grupo de pacientes com o perfil descrito.

Ainda, o risco para o desenvolvimento da arritmia é 2,12 maior no grupo que não utiliza medicamento, quando comparado com o grupo que utiliza.

Percebe-se uma tendência em desencadear arritmia os pacientes que apresentaram distúrbios do potássio, cálcio e não uso de medicamentos, possivelmente em decorrência do desequilíbrio entre as concentrações iônicas dos meios intra e extracelulares.

Na Tabela 4 temos a distribuição do momento de ocorrência da arritmia ventricular e supraventricular. Verifica-se que a maioria dos pacientes não apresentou arritmia ventricular (17 casos, 48,6\%), enquanto 31,4\% tiveram fora da diálise (11 casos), $14,3 \%$ tiveram tanto na diálise como fora dela (5 casos) e 5,7\% tiveram apenas durante a diálise (2 casos). Para a arritmia supraventricular observa-se que 40,0\% não tiveram (14 casos), cerca de 42,9\% desenvolveram arritmia supraventricular fora da diálise (15 casos), 14,3\% teve tanto na diálise como fora dela (14,3\%) e apenas $2,9 \%$ desenvolveram na diálise. O teste de comparação de distribuição não foi significativo ( $p$-valor $=0,724$ ), indicando que a ocorrência da arritmia ventricular e a arritmia supraventricular ocorre de forma semelhante. 
Tabela 4. Distribuição do momento de ocorrência das arritmias.

\begin{tabular}{lcc}
\hline \multirow{2}{*}{ Horário de ocorrência } & \multicolumn{2}{c}{ Tipo de arritmia } \\
\cline { 2 - 3 } & $\begin{array}{c}\text { Arritmias } \\
\text { Ventriculares }\end{array}$ & $\begin{array}{c}\text { Arritmias } \\
\text { Supra- } \\
\text { ventriculares }\end{array}$ \\
\hline 0 - Não teve & $17(48,6 \%)$ & $14(40,0 \%)$ \\
1 - Teve na diálise & $2(5,7 \%)$ & $1(2,9 \%)$ \\
2 - Fora da diálise & $11(31,4 \%)$ & $15(42,9 \%)$ \\
1 e 2 - Durante e antes da diálise & $5(14,3 \%)$ & $5(14,3 \%)$ \\
\multicolumn{1}{c}{ TOTAL } & $35(100 \%)$ & $35(100 \%)$ \\
\hline
\end{tabular}

Fonte: Autores (2021).

\section{Discussão}

O Holter ECG, dispositivo utilizado nesse estudo, realizado por um período de 24 horas, é amplamente utilizado como medida não invasiva para a detecção de arritmia cardíaca, geralmente não encontrada no ECG usual. Além disso, esse método pode avaliar alterações dos batimentos cardíacos, como a variabilidade da frequência cardíaca (Kida et al., 2017).

Os achados evidenciam significância estatística entre a idade e a probabilidade de desenvolvimento de arritmias pelos pacientes. Também observa-se a considerável prevalência de hipertensão arterial. Estudos apontam que diversos fatores agravam as arritmias cardíacas em pacientes com DRC em hemodiálise e aumentam o risco de morte súbita cardíaca. Entre esses fatores destacam-se: idade, hipertrofia ventricular esquerda (HVE), Diabetes Mellitus, cardiopatia pré-existente, hipertensão arterial e outros (Akoum et al., 2019).

Como observado na Tabela 1, os dados absolutos apontam maior prevalência da DRC em homens, não negros e idade acima de 50 anos (57,2\%). Isso corrobora com alguns estudos que apontam a mesma tendência (Moura et al., 2015; Pinho et al., 2015). A longevidade da população é um dos fatores que contribuem com o aumento da prevalência da DRC (Collins et al., 2015).

A eficiência dialítica pode ser avaliada pelo $\mathrm{Kt} / \mathrm{V}$, variável relacionada à depuração do dialisador, ao tempo de diálise e ao volume de distribuição do soluto (uréia) no paciente. Portanto, o Kt/V estabelece a prescrição de uma diálise adequada e é importante para o controle da hipertensão arterial. Sabe-se que valores de Kt/V superiores a 1,2 estão relacionados a morbidade e mortalidade reduzidas em pacientes com DRC em hemodiálise (Teixeira et al., 2015).

A hipertensão arterial, frequentemente encontrada em pacientes em hemodiálise, e o equivalente a 77,1\% dos pacientes no presente estudo, é relatada como o fator mais importante para distúrbios cerebrovasculares, pois pode induzir distúrbios estruturais ou funcionais no miocárdio, levando à arritmia. Portanto, a HAS pode aumentar o risco de morte súbita dos pacientem em terapia dialítica (Kida et al., 2017).

Outro aspecto observado, foi o maior risco de desenvolver arritmia para os indivíduos que não utilizava medicamento. Isso pode estar relacionado pelo fato de muitos pacientes serem subdiagnosticados quanto à necessidade de utilizarem esses medicamentos, mostrando a importância do registro eletrocardiográfico (Holter) nesses pacientes (Yuyun et al., 2020).

Os dados (Tabela 3) corroboram com outros achados que relacionam os níveis inadequados de potássio e cálcio ao risco de arritmia e morte em pacientes com DRC em hemodiálise (Teixeira et al., 2015; Bonato \& Canziani, 2017). Estudos evidenciam que do ponto de vista cardiológico, as arritmias cardíacas podem ser desencadeadas ou facilitadas por esses distúrbios hidroeletrolíticos, caracterizados por variação dos níveis normais de eletrólitos (como o cálcio e o potássio) mesmo em corações estruturalmente normais. Níveis anormais de cálcio podem causar intercorrências cardíacas como o prolongamento ou desnivelamento do segmento ST ou prolongamento do intervalo QT, caracterizando arritmias (Souza et al., 2020).

Em modelos experimentais clínicos, os distúrbios envolvendo os níveis de potássio e cálcio são capazes de 
desencadear arritmias, possivelmente, em virtude do desequilíbrio entre as concentrações iônicas dos meios intra e extracelulares (Bonato \& Canziani, 2017).

O tempo de sobrevida de pacientes renais crônicos em hemodiálise depende de fatores como: sexo, idade, índice de adequação da diálise $(\mathrm{Kt} / \mathrm{V})$, hemoglobina $(\mathrm{Hb})$, albumina, produto cálcio x fósforo $(\mathrm{Ca} \times \mathrm{P})$ e paratormônio $(\mathrm{PTH})$, entre outros, como supramencionado. Portanto, a avaliação desses parâmetros permite obter informações a respeito do riso de morte dos pacientes (Teixeira et al., 2015).

Estudos apontam que os distúrbios eletrolíticos no potássio e cálcio são comuns em pacientes com DRC, mesmo em estágios moderados, e podem levar a arritmias, aumentando o risco de morte súbita cardíaca (Akoum et al., 2019; Karaboyas et al., 2017).

Percebe-se na tabela 4 que não houve diferença entre os grupos, quanto às arritmias ventriculares e supraventriculares, visto que não houve significância estatística. Alguns estudos apontam maior tendência na ocorrência de arritmias ventriculares durante a diálise, em comparação com as arritmias supraventriculares (Turakhia et al., 2018; Lullo et al., 2016). A ausência de significância estatística neste estudo pode estar relacionado com o número reduzido de indivíduos.

Os achados apontam que a fisiopatologia das arritmias na população com DRC é multifatorial. Portanto, Além de fatores envolvendo fibrilação, processos inflamatórios, teciduais e doenças pré-existentes como Diabetes Mellitus e Hipertensão Arterial Sistêmica, as arritmias ocorrem devido a perturbações eletrolíticas no potássio, cálcio e fosfato.

\section{Considerações Finais}

Diante das observações sucedidas, foi possível constatar que houve maior prevalência de DRC em indivíduos do sexo masculino e que se consideraram não negros. Não houve diferença estatística entre a ocorrência as arritmias ventriculares e supraventriculares em pacientes com DRC em hemodiálise, apontando a mesma prevalência entre esses distúrbios nos indivíduos avaliados.

Níveis inadequados de potássio e cálcio aumentam a prevalência de arritmia e risco de morte em pacientes com DRC em hemodiálise. Além disso, a idade mostrou-se como um importante fator e risco para a ocorrência de arritmias. Nessa diretriz, torna-se evidente que a maior prevalência de arritmias está associada a níveis inadequados de potássio, cálcio e aos portadores de HAS. Durante a diálise, houve maior incidência de arritmia ventricular. Todos esses fatores contribuem com o risco de morte súbita cardíaca em paciente com DRC dialítica.

Diante desses achados, ressalta-se a importância de estudos com uma maior população amostral, bem como maior variabilidade de raça e/ou variabilidade filogenética, de modo a investigar a interferência desses fatores com maior risco cardiovascular diante da terapia dialítica substitutiva. Além disso, sabe-se que o tempo de hemodiálise está diretamente relacionado com o risco cardiovascular (Balbino et al., 2018). Portanto, torna-se relevante a investigação da correlação entre tempo de hemodiálise e os parâmetros avaliados neste estudo, de modo a esclarecer as variáveis envolvidas na susceptibilidade dos pacientes renais crônicos ao desenvolvimento de intercorrências cardiovasculares, como as arritmias cardíacas.

\section{Referências}

Akoum, N., Zelnick, L. R., De Boer, I. H., Hirsch, I. B., Trence, D., Henry, C., Robinson, N. \& Bansal, N. (2019). Rates of Cardiac Rhythm Abnormalities in Patients with CKD and Diabetes. Clinical Journal of the American Society of Nephrology, 14, 549-556.

Almeida, A. M. \& Rabinovich, E. P. Vivências de familiares de pessoas em hemodiálise durante a pandemia do novo corona vírus (COVID-19). (2020). Research, Society and Development, 9(8), e887986661.

Balbino, K. P. Hermsdorf, H. H. M. \& a Bressan, J. (2018). Polymorphism related to cardiovascular risk in hemodialysis subjects: a systematic review. Braz. J. Nephrol, 40(2):179-192. 
Bauer, C., Melamed, M. L. \& Hostetter, T. H. Staging of chronic kidney disease: time for a course correction. (2008). Journal of the American Society of Nephrology, 19(5), 844-846.

Bignotto, L. H., Kallás, M. E., Djouki, R. J., Sassaki, M. M., Voss, G. O., Soto, C. L., Frattini, F. \& Medeiros, F. S. (2012). Achados eletrocardiográficos em pacientes com doença renal crônica em hemodiálise. Jornal Brasileiro de Nefrologia, 34(3), $235-242$.

Bonato, F. \& Canziani, M. (2017). Arritmia ventricular em pacientes com doença renal crônica. Jornal Brasileiro de Nefrologia, 39 (2), 186-195.

Collins A. J., Foley, R. N., Gilbertson, D. T. \& Chen, S. (2015). United States Renal Data System public health surveillance of chronic kidney disease and end-stage renal disease. Kidney International Supplements, 5(1), 2-7.

Donfrancesco, C., Palleschi, S., Palmieri, L., Rossi, B., Noce, C., Pannozzo, F., Stopo, B., Tripepi, G., Zoccali, C. \& Giampaoli, S. (2013). Estimated glomerular filtration rate, all-cause mortality and cardiovascular diseases incidence in a low risk population: the MATISS study. PLoS One, 8(10), e78475.

Hsueh, C., Chen, N. X., Lin, S., Chen, P., Gattone, V. H., Allen, M. R., Fishbein, M. C. \& Moe, S. M. (2014). JASN, 25(12), $2812-2821$.

Johnson, R. J., Nakagawa, T., Jalal, D., Sánchez-Lozada, L. G., Kang, D. H., Ritz, E. Uric acid and chronic kidney disease: which is chasing which? (2013). Nephrol Dial Transplant, 28(9), 2221-2228.

Karaboyas A, et al. (2017). Dialysate potassium, serum potassium, mortality, and arrhythmia events in hemodialysis: results from the Dialysis Outcomes and Practice Patterns Study (DOPPS). American Journal of Kidney Diseases, 69, 266-277.

Kida, N., Tsubakihara, Y., Kida, H., Ageta, S., Arai, M., Hamada, Y. \& Matsuura, N. (2017). Usefulness of measurement of heart rate variability by holter ECG in hemodialysis patients. BMC Nephrology., 18(8), 1-7.

Kidney Disease: Improving Global Outcomes (KDIGO). CKD Work Group. KDIGO 2012, Clinical Practice Guideline for the Evaluation and Management of Chronic Kidney Disease. Kidney Int (Suppl). 3: 1-150.

Lullo L. D, Rivera R, Barbera V, Bellasi A, Cozzolino M, Russo D, De Pascalis A, Banerjee D, Floccari F, \& Ronco C. (2016). Sudden cardiac death and chronic kidney disease: From pathophysiology to treatment strategies. International Journal of Cardiology, $217,16-27$.

Marinho, A. W., Penha, A. P., Silva, M. T. \& Galvão, T. F. Prevalência de doença renal crônica em adultos no Brasil: revisão sistemática da literatura. (2017). Cadernos Saúde Coletiva, 25(3), 379-388.

Moura, L., Andrade, S., Malta, D., Pereira, C. \& Passos, J. (2015). Prevalência de autorrelato de diagnóstico médico de doença renal crônica no Brasil: Pesquisa Nacional de Saúde, 2013. Revista Brasileira de Epidemiologia, 18(2), 181-191.

Neves, P. D., Sesso, R. C., Thomé, F. S., Lugon, J. R. \& Nascimento, M. Censo Brasileiro de Diálise: análise de dados da década 2009-2018. (2020). Jornal Brasileiro de Nefrologia, 42(2), 191-200.

Pinho, N., Silva, G. \& Pierin, A. (2015). Prevalência e fatores associados à doença renal crônica em pacientes internados em um hospital universitário na cidade de São Paulo. Jornal Brasileiro de Nefrologia, 37(1), 91-97.

R Core Team. R. (2013). A language and environment for statistical computing. R Foundation for Statistical Computing., Vienna, Austria. http://www.Rproject.org/.

SBN - Sociedade Brasileira de Nefrologia. Censo Brasileiro de Diálise: 2009-2018. SBN. http://www.sbn.org.br.

Souza, M. L. et al. (2020). Revisão da literatura sobre os principais distúrbios hidroeletrolíticos. Revista Saberes da Faculdade São Paulo, 14(1).

Teixeira, F., Lopes, M., Silva, G. \& Santos, R. (2015). Sobrevida de pacientes em hemodiálise em um hospital universitário. Jornal Brasileiro de Nefrologia, 37(1), 64-71.

Turakhia M, et al. (2018). Chronic kidney disease and arrhythmias: conclusions froma Kidney Disease: Improving Global Outcomes (KDIGO) Controversies Conference. European Heart Journal, 39, 2314-2325.

Verde, E., Pérez de Prado, A., López-Gómez, J. M., Quiroga, B., Goicoechea, M., García-Prieto, A., Torres, E., Reque, J. \& Luño, J. (2016). Asymptomatic intradialytic supraventricular arrhythmias and adverse outcomes in patients on hemodialysis. Clinical Journal of the American Society of Nephrology, 11, $2210-2217$

Yuyun, M. et al. (2020). A Systematic Review of the Spectrum of Cardiac Arrhythmias in Sub-Saharan Africa. Global Heart, $15(1), 37$. 